\title{
BMJ Open Major cardiovascular events in patients presenting with acute stroke: a 5-year follow-up study in patients who had ischaemic stroke and stroke mimics
}

\author{
Salman Al Jerdi (D) , ${ }^{1}$ Naveed Akhtar, ${ }^{2}$ Ziyad Mahfoud, ${ }^{3}$ Saadat Kamran, ${ }^{4}$ \\ Ashfaq Shuaib (1)
}

To cite: Al Jerdi S, Akhtar N, Mahfoud Z, et al. Major cardiovascular events in patients presenting with acute stroke: a 5-year followup study in patients who had ischaemic stroke and stroke mimics. BMJ Open 2022;12:e053059. doi:10.1136/ bmjopen-2021-053059

- Prepublication history for this paper is available online. To view these files, please visit the journal online (http://dx.doi. org/10.1136/bmjopen-2021053059).

Received 05 May 2021 Accepted 20 December 2021

Check for updates

(c) Author(s) (or their employer(s)) 2022. Re-use permitted under CC BY-NC. No commercial re-use. See rights and permissions. Published by BMJ.

${ }^{1}$ Department of Neurology, Weill Cornell Medicine - Qatar, Doha, Qatar

${ }^{2}$ Neuroscience Institute, Hamad Medical Corp, Doha, Qatar

${ }^{3}$ Department of Medical

Education, Weill Cornell

Medicine - Qatar, Doha, Qatar

${ }^{4}$ Neuroscience Institute, Hamad Medical Corporation, Doha, Ad Dawhah, Qatar

${ }^{5}$ Faculty of Medicine and Dentistry, University of Alberta, Edmonton, Alberta, Canada

Correspondence to

Dr Ashfaq Shuaib;

ashfaq.shuaib@ualberta.ca

\section{ABSTRACT}

Objectives The long-term acute stroke outcome has not been well studied in the Middle-Eastern population. The primary objective of our study is to compare the longterm outcome of acute ischaemic stroke (IS) with/without previous cerebrovascular/cardiovascular disease (CVD) to stroke mimics (SM) with CVD.

Settings and participants The Qatar stroke database was reviewed for IS and SM admissions in Qatari Nationals between 2013 and 2019.

Outcomes Patients were prospectively assessed for development of recurrent stroke, myocardial infarction or death. Frequency of major cardiovascular events (MACEs) were compared between patients with or without a previous CVD.

Results There were 1114 stroke admissions (633 IS (prior CVD 211/18.9\%), 481 SM (prior CVD 159/14.3\%)). Patients with IS/CVD were significantly older versus others (IS/CVD: 68.3 $\pm 12.2 ;$ IS/no CVD: 63.3 \pm 14.4 ; SM/CVD: 67.6 $\pm 13.1 ;$ SM/no CVD: $52.4 \pm 17.9 . p<0.0001)$. Vascular risk factors were significantly higher in patients with IS and SM with previous CVD. Functional recovery (90-day mRS 0-2) was significantly better in SM/no CVD (IS/CVD: 55.0\%; IS/no CVD: 64.2\%; SM/CVD 59.7\%; SM/no CVD: $88.8 \%$. p <0.001). MACE occurred in 36\% (76/211) IS/ CVD, 24.9\% (105/422) IS/no CVD, 22.0\% (35/179) SM/ CVD and only $6.8 \%$ (22/322) SM/no CVD. MACE occurred mostly during the first year of follow-up. Mortality 90 days was significantly higher in IS/CVD (IS/CVD 36\%; IS/no CVD 24.9\%; SM/CVD: 22\%; SM/no CVD: 6.8\%. p<0.0001). Conclusions Prior CVD significantly increases the risk of MACE and early mortality in IS or SM patients. Age, male gender, obesity, atrial fibrillation and admission National Institute of Health Stroke Scale also increases risk of MACE during follow-up. Hence, aggressive vascular risk factor modification is needed even in patients with SM.

\section{INTRODUCTION}

Patients who suffer from an acute stroke (AS) are at an increased risk of major atherosclerotic cardiovascular events (MACEs) during follow-up. The risk of MACE is higher in the initial year following the stroke, especially in individuals with a previous history of cardio/cerebrovascular disease (CVD). ${ }^{1}$ This
Strengths and limitations of this study

- This is the first study in the Middle Eastern population that evaluates for the risk of development of major cardiovascular events (MACEs) in patients presenting with an acute stroke or stroke mimics. The MACE risk was evaluated in patients with and without previous history of cerebro/cardiovascular events (CVD).

- The presence of a previous history of CVD was an important factor for MACE during follow-up. This was evident in patients who had ischaemic stroke or stroke mimics. The risk of MACE was lowest in patients with no previous history of CVD.

- The small number of patients from a single region of the Middle East is a limitation of the study that may require confirmation from other regions.

risk has decreased significantly during the last two decades, which is largely attributed to improved management of vascular risk factors. $^{2}$

There are very few studies evaluating the risk of MACE in stroke mimics $(\mathrm{SM}){ }^{3}$ Similar to AS, the risk of vascular events during follow-up in SM also depends on the presence of underlying preexisting vascular disease and is higher in medical mimics compared with functional mimics. ${ }^{4}$ Additionally, in medical mimics, a higher cardiovascular event rate may occur in patients who experience 'recrudescence of symptoms' in the presence of a previous history of stroke. ${ }^{5}$

The long-term prognosis in patients who had an ischaemic stroke (IS) in the Middle East has received very little attention. We have previously studied the stroke subtypes, risk factors and early prognosis in patients who had an $\mathrm{AS}^{6-8}{ }^{6} \mathrm{SM}^{9}$ and poststroke depression in Qatar. ${ }^{10}$ We have also evaluated the longerterm prognosis and MACE in the ethnically distinct Qatari population. During the early 
recovery phase following an AS, recovery is slower in women and mortality is higher compared with men. However, during long-term follow-up, men tend to have higher MACE, especially in the presence of previous coronary artery disease. ${ }^{11}$

The primary objective of the present research is to evaluate the short-term and long-term prognosis of AS and stroke mimic patients in an ethnically distinct Arab population in Qatar. We investigated the outcomes in patients who had an acute IS in the presence and absence of previous CVD and similarly in SM with and without previous CVD. We selected patients with SM without a history of CVD both to serve as a control group and to provide insight about the risk of MACE in this relatively understudied population.

\section{PATIENTS AND METHODS}

\section{Study population}

All AS and stroke mimic patients admitted to the Hamad General Hospital (HGH) from January 2014 to February 2019 were evaluated for the study. Patients or the public were not involved in the design, or conduct, or reporting, or dissemination plans of our research. SM included both medical and functional mimics. The information was obtained from the discharge diagnosis after patient completed their in-hospital evaluation. Clinical information including ethnicity, risk factors, clinical presentation and course in hospitalisation was entered prospectively in a database. We also documented all investigations, diagnosis and complications as previously published. ${ }^{67}$ The hospital has a dedicated stroke ward, admits $98 \%$ of strokes in Qatar and is the only centre where AS treatment with intravenous thrombolysis and mechanical thrombectomy is offered. Patients of all ethnicities are admitted to the HGH. All patients are evaluated by stroke neurologists or stroke physicians, and their stroke is confirmed using neuroimaging (CT or MRI). For the purpose of the current research, we only focused on Qatari nationals. This allowed for accurate follow-up information on the study subjects. Non-nationals who had a stroke typically travel to their home countries for further treatment or recovery, thus limiting accurate follow-up data. Patients with a final diagnosis of IS and SM were reviewed for this study. The prehospital modified Rankin Scale (mRS), National Institutes of Health Stroke Scale (NIHSS) and the mRS at discharge and 90-days follow-up were documented. We reviewed the state-wide Cerner medical records of the patients to document any vascular complications following discharge.

We did not include patients with transient ischaemic attacks (TIAs) as the diagnosis is not always clear. There is risk that SM may be wrongly classified as TIAs or vice versa. We also did not review records of patients admitted with intracranial haemorrhage (ICH) as the mechanisms for most patients are not related to atherosclerosis.

\section{Study outcomes}

The patients were categorised into four categories: ischaemic stroke with previous evidence of either stroke or cardiac disease (CVD); ischaemic stroke with no previous history of CVD; stroke mimic with previous CVD and stroke mimic with no previous CVD. We reviewed the severity of symptoms, hospital course, risk of medical complications in hospital and mortality during hospitalisation and in the 90 days following discharge. The primary shortterm outcome was mRS score at 90 days. The primary long-term outcome was occurrence of MACE. MACE was defined as cardiovascular mortality, all-cause mortality, fatal or non-fatal MI, recurrent stroke, congestive heart failure and revascularisation procedure (CABG or PCI), after the index event during follow-up.

\section{Patient and public involvement \\ No patient involvement.}

\section{Statistical methods}

Descriptive and inferential statistics were used to characterise the study sample. Descriptive results (including graphical displays) for all quantitative variables (eg, age) are presented as the mean $\pm \mathrm{SD}$ for normally distributed data or median with IQR for data not normally distributed. Bivariate analysis was performed using independent sample t-test and the Mann Whitney U-test to compare the average for all quantitative variables (eg, age) between stroke subtypes (IS vs SM), wherever appropriate, while the Pearson $\chi^{2}$ test or Fisher's exact test as appropriate were used in comparing all the qualitative variables (eg, presence or absence of CVD) between IS and SM. The Kaplan-Meier survival analysis was applied to compare differences in the cumulative incidence of MACE in 5 years among the four groups. The log rank test (Mantel-Cox Method) was used to test the null hypothesis that there was no difference between the four groups in the probability of an event at any time point. To understand the effect of age and other risk factors, HRs for MACE were determined by multivariate Cox proportional hazards regression analyses with data presented as HR (compared with the lowest quartile) with 95\% CIs. The statistical tests were performed in IBM SPSS Statistics V.26 (IBM, Armonk, USA).

\section{RESULTS}

\section{Demographics}

There were 8979 patients admitted with an AS to the HGH during the study period. Of these, 1515 were Qatari nationals. After excluding patients with ICH $(n=179)$, cerebral venous thrombosis $(n=8)$ and TIAs $(n=212)$, we were left with 1114 patients who had an IS and SM for analysis for our study (mean age $61.7 \pm 16.2$; male: 610 (54.8), female: $504(45.2))$. These included IS with previous CVD: 211 (18.9\%); IS with no previous CVD $422(37.9 \%)$, SM with previous CVD $159(14.3 \%)$ and SM with no previous CVD: 322 (28.9\%). SM with no 
Table 1 The demographics, clinical features and short-term prognosis are shown for the four cohorts of patients

\begin{tabular}{|c|c|c|c|c|c|c|}
\hline $\begin{array}{l}\text { Characteristic or } \\
\text { investigation }\end{array}$ & Total $(n=1114)$ & $\begin{array}{l}\text { IS with CVD } \\
(n=211,18.9 \%)\end{array}$ & $\begin{array}{l}\text { IS without CVD } \\
(\mathrm{n}=422,37.9 \%)\end{array}$ & $\begin{array}{l}\text { SM with CVD } \\
(n=159,14.3 \%)\end{array}$ & $\begin{array}{l}\text { SM without } \\
\text { CVD } \\
\text { (n=322, 28.9\%) }\end{array}$ & $P$ value \\
\hline Age, mean, years & $61.7 \pm 16.2$ & $68.3 \pm 12.2$ & $63.3 \pm 14.4$ & $67.6 \pm 13.1$ & $52.4 \pm 17.9$ & 0.0001 \\
\hline \multicolumn{7}{|l|}{ Sex } \\
\hline Male & $610(54.8)$ & $138(65.4)$ & $234(55.5)$ & $85(53.5)$ & $153(47.5)$ & \multirow[t]{2}{*}{0.001} \\
\hline Female & $504(45.2)$ & $73(34.6)$ & $188(44.5)$ & $74(46.5)$ & $169(52.5)$ & \\
\hline Hypertension & $803(72.1)$ & $192(91.0)$ & $330(78.2)$ & $132(83.0)$ & $149(46.3)$ & 0.0001 \\
\hline Diabetes & $757(68.0)$ & $179(84.8)$ & $297(70.4)$ & $128(80.5)$ & $153(47.5)$ & 0.0001 \\
\hline Dyslipidaemia & $576(51.7)$ & $127(60.2)$ & $232(55.0)$ & $91(57.2)$ & $126(39.1)$ & 0.0001 \\
\hline $\begin{array}{l}\text { Atrial fibrillation on } \\
\text { admission }\end{array}$ & $98(8.8)$ & $39(18.5)$ & $43(10.2)$ & $13(8.2)$ & $3(0.9)$ & 0.0001 \\
\hline Active smoking & $220(19.7)$ & $42(19.9)$ & $87(20.6)$ & $28(17.6)$ & $63(19.6)$ & 0.88 \\
\hline $\begin{array}{l}\text { Obesity (BMI } \geq 30 \mathrm{~kg} / \mathrm{m}^{2} \\
(\mathrm{n}=1065)\end{array}$ & $463(43.5)$ & $82(42.9)$ & $166(42.0)$ & $60(38.0)$ & $155(48.3)$ & 0.15 \\
\hline \multicolumn{7}{|l|}{ NIHSS severity } \\
\hline Mild (NIHHS 0-4) & $821(73.7)$ & $114(54.0)$ & 263 (62.3) & $146(91.8)$ & $298(92.5)$ & \multirow[t]{3}{*}{0.0001} \\
\hline Moderate (NIHSS 5-10) & $178(16.0)$ & $56(26.5)$ & $97(23.0)$ & $7(4.4)$ & $18(5.6)$ & \\
\hline Severe (NIHSS > 10) & $115(10.3)$ & $41(19.4)$ & $62(14.7)$ & $6(3.8)$ & $6(1.9)$ & \\
\hline Mortality at 90 days & $51(4.6)$ & $22(10.4)$ & $24(5.7)$ & $3(1.9)$ & $2(0.6)$ & 0.0001 \\
\hline
\end{tabular}

BMI, body mass index; IS, ischaemic stroke; NIHSS, National Institute of Health Stroke Scale; SM, stroke mimic.

previous CVD were significantly younger than the other three groups (see table 1). The incidence of hypertension and hyperlipidaemia were highest in patients with IS and previous CVD and lowest in SM with no previous CVD. The prestroke $\mathrm{mRS}$ was higher in both IS and SM patients with previous CVD (mRS 1.1 \pm 1.7 ; IS+CVD: $1.4 \pm 1.8$; IS+no CVD: $0.8 \pm 1.5$; SM+CVD: $2.1 \pm 1.9$; SM+no CVD: $0.7 \pm 1.4$; $\mathrm{p}=0.0001$ ) (table 1). Atrial fibrillation was significantly higher in the IS groups compared with the SM groups.

We evaluated if there were differences in outcome of functional and medical mimics. Functional mimics is made up of only $17 \%(82 / 481)$ of the SM patients, while medical mimics $83 \%(399 / 481)$ of the SM patients. Medical mimics constituted $133(83.6 \%)$ of the SM patients with previous CVD and $266(82.6 \%)$ of the SM patients without previous CVD. We found no differences in the clinical presentation and short-term outcome between the functional and medical mimics.

\section{Clinical course and early prognosis following discharge}

The mode of admission to the emergency department was similar in the four groups with slightly more than $50 \%$ transferred by the ambulance service. Overall, the severity of stroke (as measured on admission NIHSS) was mild in most strokes (3.9 \pm 5.3$)$. Patients with IS and previous CVD had the most severe stroke (NIHSS of $6.3 \pm 6.3$ ) and those with SM and no previous CVD had the mildest symptoms (NIHSS 1.3 \pm 3.1 ) as shown in table 1 .

The percentage of patients admitted to the stroke unit was similar between patients with ischaemic stroke with and without CVD. Stroke-related investigations were significantly fewer in SMs compared with IS. Significantly fewer patients with SM received thrombolysis (IS+CVD: 8.5\%; IS+no CVD:10.9\%; SM+CVD: $1.3 \%$; SM+no CVD: $1.2 \% ; \mathrm{p}=0.0001)$. At the time of discharge, the percentage of patients with mRS of 0-2 (functional independence) was low in IS with previous CVD (46.7\%), IS with no previous SVD (52.8\%) and SM with previous CVD (47.2) compared with SM with no previous CVD $(82.0 \%)$. These remained similar at 90-day follow-up as well with IS and CVD (55.0\%), IS with no CVD (64.2\%) and SM with CVD (59.7\%) compared with SM with no CVD $(88.8 \%)$. There were $51(4.60 \%)$ deaths in hospital and within 90 days, of which $22(10.4 \%)$ were patients with IS and previous CVD as shown in table 1 .

\section{MACEs during follow-up}

Follow-up information was available on the electronic medical system for all patients. During follow-up, 238 $(21.4 \%)$ patients had a MACE. The incidence of MACE was highest in patients with IS and previous CVD. MACE occurred in 36\% (76/211) IS with CVD, $24.9 \%$ $(105 / 422)$ IS without CVD patients, $22.0 \%$ (35/179) SM with previous CVD and only $6.8 \%$ (22/322) SM with no CVD (see figure 1). Most events occurred during the initial year of follow-up. In patients with IS (with or without previous CVD), the most frequent MACE was recurrent stroke. In patients presenting with SMs (with or without CVD), cardiac events, including acute myocardial 
A

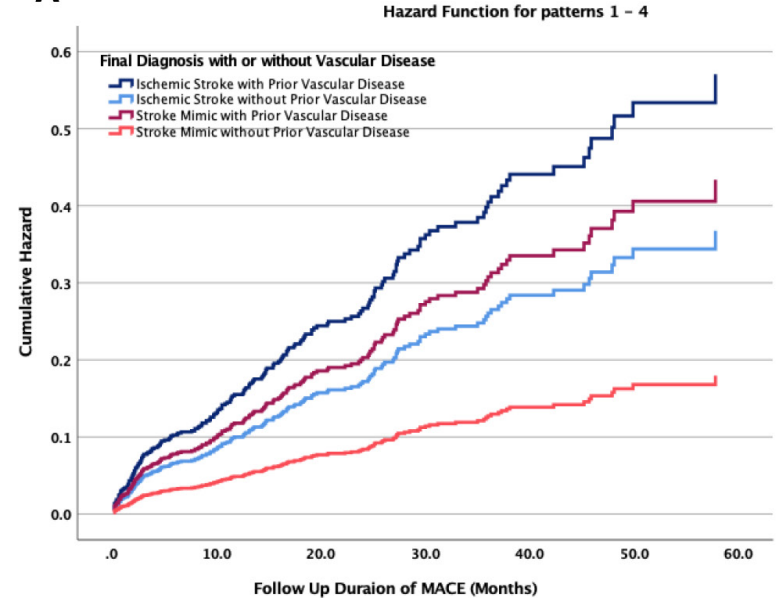

B

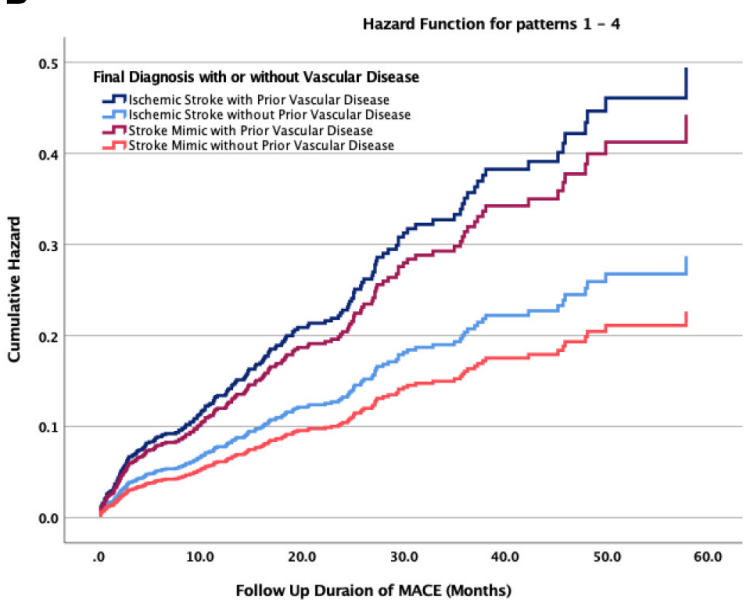

Figure 1 Hazard curves showing the 5-year probability of major cardiac adverse events (MACEs) in patients presenting as ischaemic strokes with and without prior cardiovascular disease versus stroke mimics, in Qatar (mean follow-up duration: $24.2 \pm 17.1$ months) (A) prior to controlling for discharge medications and (B) after controlling for discharge medications.

infarction were more frequent than ischaemic stroke (see table 2).

Multivariate analysis showed the incidence of MACE was associated with increasing age, sex (male), obesity, presence of atrial fibrillation on admission and National Institute of Health Stroke Scale (NIHSS) on admission as shown in table 3 .

Overall, compared with SM patient without CVD, both patients with IS or SM that had CVD had an increased risk of MACE. Although IS patients without CVD were initially found to also have an increased risk of MACE, this lost its statistical significance difference when discharge medications where factored in.

\section{DISCUSSION}

In this cohort of 1114 patients who had an AS, we made several important observations. Patients who had an IS with a previous history of CVD were most likely to experience severe symptoms at presentation and were significantly more likely to die during hospitalisation and the 90 days following discharge.

Table 2 Major cardiovascular events (MACEs) during follow-up in the four groups

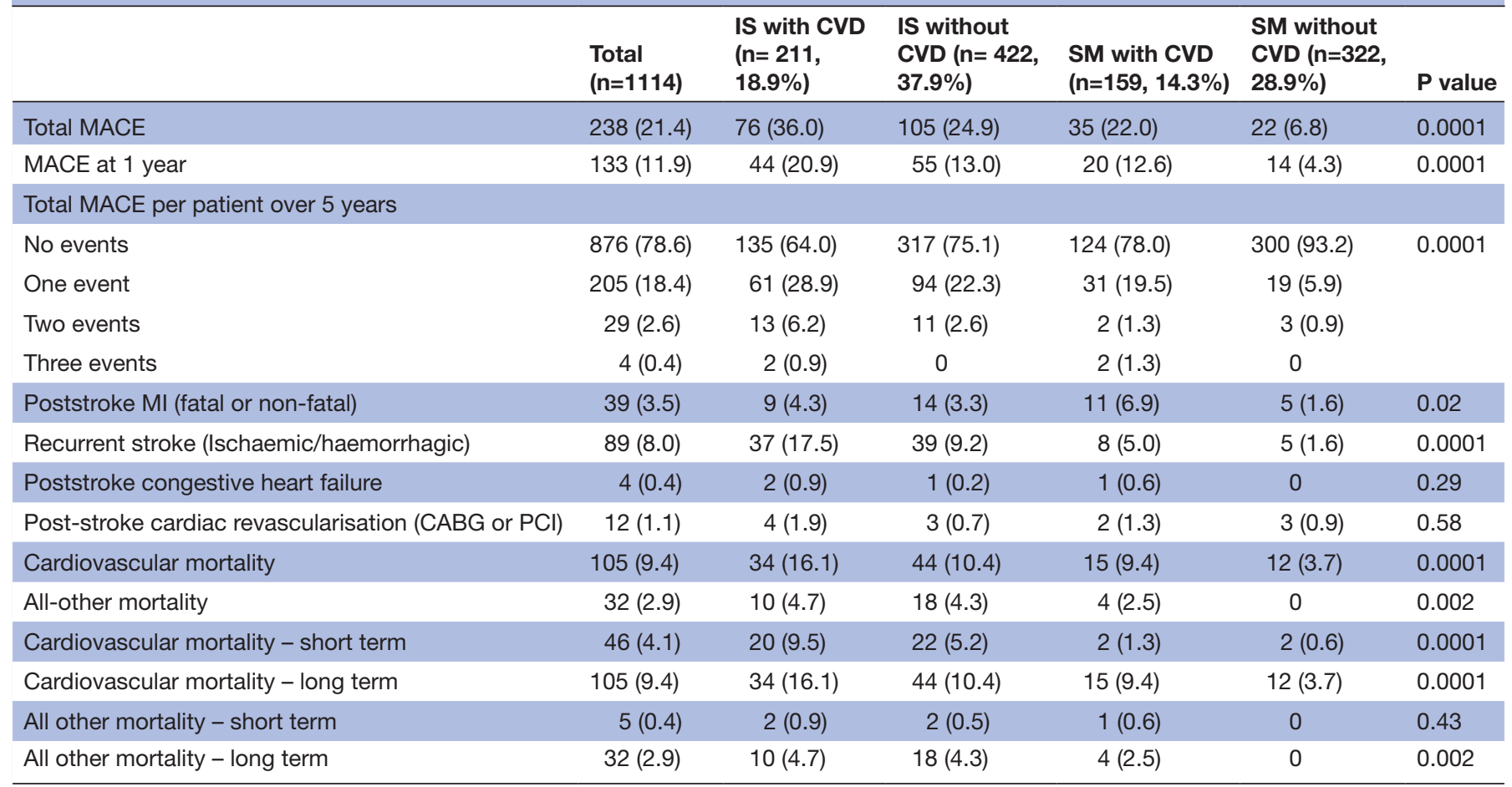

The type of MACE events and time of the vascular outcome events are shown in the table.

$\mathrm{CABG}$, coronary artery bypass grafting; $\mathrm{MI}$, myocardial infarction; $\mathrm{PCl}$, percutaneous intervention. 
Table 3 Cox regression analysis of covariates associated with occurrence of major cardiac adverse event (MACE) stratified for diagnosis (stroke mimics vs ischaemic stroke - with or without cardiovascular history) in patients presenting as acute stroke from Qatar

\begin{tabular}{|c|c|c|c|c|}
\hline \multirow[b]{2}{*}{ Characteristic } & \multirow[b]{2}{*}{ AHR } & \multicolumn{2}{|l|}{$95.0 \% \mathrm{Cl}$} & \multirow[b]{2}{*}{$P$ value } \\
\hline & & Lower $\mathbf{C l}$ & Upper Cl & \\
\hline \multicolumn{5}{|l|}{ Stroke mimic without prior vascular disease } \\
\hline Ischaemic stroke without prior vascular disease & 1.268 & 0.657 & 2.445 & 0.479 \\
\hline Stroke mimic with prior vascular disease & 1.954 & 1.065 & 3.585 & 0.031 \\
\hline Female & 0.495 & 0.351 & 0.698 & 0.0001 \\
\hline AF on admission & 1.830 & 1.150 & 2.913 & 0.011 \\
\hline Obesity (BMI $\geq 30)$ & 0.728 & 0.536 & 0.989 & 0.042 \\
\hline NIHSS on admission & 1.065 & 1.041 & 1.089 & 0.000 \\
\hline
\end{tabular}

AF, Atril Fibrillation; AHR, adjusted HR; BMI, body mass index; NIHSS, National Institute of Health Stroke Scale.

More than $20 \%$ of patients developed a MACE even during follow-up. Whereas the long-term prognosis was relatively benign in patients with $\mathrm{SM}$ with no previous history of CVD, the risk of MACE was high in those with $\mathrm{SM}$ with previous CVD. The distribution of functional versus medical mimics in both groups was not statistically significantly different.

Patients who had an IS were more likely to experience recurrent stroke as their MACE event, while patients with SM were more likely to have a cardiac MACE. The incidence of MACE was highest during the initial year following the index event.

Two recent studies followed AS patients for MACE events. $^{2}{ }^{11}$ In the study from Oxford, MACE events were documented in $22 \%$ of patients and were more frequent in patients over the age of 75 years. Similar to our study, MACE was more frequent in patients with pre-existing CVD versus no history of CVD (22\% vs $7 \%$ ), and recurrent stroke was the most common event. The study from Ontario, Canada, evaluated the risk of cardiac events and compared it with age-matched controls (propensitymatched individuals with no history of stroke). The risk of MACE during follow-up was four times higher in patients who had a stroke and was highest early following the AS. ${ }^{12}$

The frequency of SM in the Qatari stroke population in our study is around $36 \%$ and appears similar to previously published reports. ${ }^{1-3}$ Similar to reports from Europe and North America, medical mimics account for the majority of patients. In our study, many SM patients had a previous history of stroke $(22 \%, 106 / 481)$. Our study is the first report highlighting the importance of MACE during follow-up in SM.

We were able to show that MACE events are high in SM, especially patients with previous history of CVD. The risk of recurrent events is strongly related to the presence or absence of pre-existing CVD. MACE was noted in $22 \%$ of SM patients with previous CVD and less than $7 \%$ of SM with no history of CVD. Thus, recognising vascular risk factors in SM is therefore important and prevention strategies should be aggressively pursued to reduce the risk of recurrence. An alternate possibility for the high rates of MACE that cannot be excluded is the possibility of a missed diagnosis of an AS (with negative imaging), resulting in a diagnosis of stroke mimic. With the high frequency of small vessel disease in our population, we cannot rule out this possibility.

The strength of our study is the comprehensive nature of our prospective database and the ability to capture all events in a nationwide electronic medical record system. All patients seen in the hospital acutely with stroke-like symptoms were followed, and all MACE events were recorded.

The study has limitations. First, the number of patients is relatively small, and we were not able to verify the cause of death in most patients as autopsy is rarely performed in Qatar. The number is further limited by excluding patients with TIA, potentially influencing the analysis. Second, the study population is from Qatar, thus limiting generalisability. Third, we were unable to verify adherence to prescribed medical therapy as the patients were not seen in the clinic unless they had new events. Fourth, fewer brain, vascular and cardiac imaging studies were performed in women compared with men. We identify this as a concerning trend and cannot provide a clear explanation for this discrepancy. Finally, MACE data were collected from electronic medical records, and individual cases were not verified by the investigators.

Acknowledgements We acknowledge the assistance of all involved physicians, nurses and staff of the Stroke Team in Hamad Medical Corporation (HMC). We would also like to thank Ms Reny Francis (HMC) and Kath McKenzie (University of Alberta) for her editorial assistance and supportive care.

Contributors Concept, design and draft: NA, AS and SAJ; acquisition, analysis, interpretation of data, technical and administrative support: SAJ. Ashfaq Shuaib accepts full responsibility for the work as guarantor and/or the conduct of the study, had access to the data, and controlled the decision to publish. 
Funding We received a generous funding from HMC for the grant 16016/16, a quality improvement study.

Competing interests None declared.

Patient and public involvement Patients and/or the public were not involved in the design, or conduct, or reporting, or dissemination plans of this research.

Patient consent for publication Not applicable.

Ethics approval The study was approved by the Institutional Review Board, Hamad Medical Corporation at the Medical Research Centre (MRC-16016/16).

Provenance and peer review Not commissioned; externally peer reviewed.

Data availability statement Data are available on reasonable request. All data relevant to the study are included in the article or uploaded as supplemental information. No additional data available.

Open access This is an open access article distributed in accordance with the Creative Commons Attribution Non Commercial (CC BY-NC 4.0) license, which permits others to distribute, remix, adapt, build upon this work non-commercially, and license their derivative works on different terms, provided the original work is properly cited, appropriate credit is given, any changes made indicated, and the use is non-commercial. See: http://creativecommons.org/licenses/by-nc/4.0/.

\section{ORCID iDs}

Salman Al Jerdi http://orcid.org/0000-0003-3495-0365

Ashfaq Shuaib http://orcid.org/0000-0002-3380-7068

\section{REFERENCES}

1 Boulanger M, Li L, Lyons S, et al. Effect of coexisting vascular disease on long-term risk of recurrent events after TIA or stroke. Neurology 2019;93:e695-707.
2 Boulanger M, Béjot Y, Rothwell PM, et al. Long-Term risk of myocardial infarction compared to recurrent stroke after transient ischemic attack and ischemic stroke: systematic review and metaanalysis. J Am Heart Assoc 2018;7:e007267.

3 Dutta D, Bowen E, Foy C. Four-year follow-up of transient ischemic attacks, strokes, and mimics. Stroke 2015;46:1227-32.

4 Gelauff J, Stone J, Edwards M, et al. The prognosis of functional (psychogenic) motor symptoms: a systematic review. J Neurol Neurosurg Psychiatry 2014;85:220-6.

5 Popkirov S, Stone J, Buchan AM. Functional neurological disorder: a common and treatable stroke mimic. Stroke 2020;51:1629-35.

6 Akhtar N, Kamran S, Singh R, et al. Beneficial effects of implementing stroke protocols require establishment of a geographically distinct unit. Stroke 2015;46:3494-501.

7 Akhtar N, Salam A, Kamran S, et al. Ethnic variation in acute cerebrovascular disease: analysis from the Qatar stroke Registry. Eur Stroke J 2016;1:231-41.

8 Akhtar N, Kamran S, Singh R, et al. The impact of diabetes on outcomes after acute ischemic stroke: a prospective observational study. J Stroke Cerebrovasc Dis 2019;28:619-26.

9 Wilkins SS, Bourke P, Salam A, et al. Functional stroke mimics: incidence and characteristics at a primary stroke center in the middle East. Psychosom Med 2018;80:416-21.

10 Wilkins SS, Akhtar N, Salam A, et al. Acute post stroke depression at a primary stroke center in the middle East. PLOS One 2018;13:e0208708.

11 Akhtar N, Kate M, Kamran S, et al. Sex-specific differences in shortterm and long-term outcomes in acute stroke patients from Qatar. Eur Neurol 2020;83:154-61.

12 Sposato LA, Lam M, Allen B, et al. First-ever ischemic stroke and increased risk of incident heart disease in older adults. Neurology 2020;94:e1559-70. 